\title{
ERRATUM
}

\section{Performance of the Gen-Probe Transcription-Mediated Amplification Research Assay Compared to That of a Multitarget Real-Time PCR for Mycoplasma genitalium Detection}

\author{
Justin Hardick, ${ }^{1}$ Julie Giles, ${ }^{1}$ Andrew Hardick, ${ }^{1}$ Yu-Hsiang Hsieh, ${ }^{2}$ Thomas Quinn, ${ }^{1,3}$ \\ and Charlotte Gaydos ${ }^{1}$
}
Division of Infectious Diseases Johns Hopkins University School of Medicine, Baltimore, Maryland ${ }^{1}$; Johns Hopkins University Department of Emergency Medicine, Baltimore, Maryland ${ }^{2}$; and National Institute of Allergy and Infectious Diseases, National Institutes of Health, Bethesda, Maryland ${ }^{3}$

Volume 44, no. 4, p. 1236-1240, 2006. Page 1236: The article title and affiliation line should read as given above.

Page 1236, abstract, line 9: "transmission" should read "transcription."

Page 1236, column 2, line 12: "transmission" should read "transcription." 